\title{
Effects of Thermal Radiation on Mixed Convection Flow of a Micropolar Fluid from an Unsteady Stretching Surface with Viscous Dissipation and Heat Generation/Absorption
}

\author{
Khilap Singh and Manoj Kumar \\ Department of Mathematics, Statistics and Computer Science, G.B. Pant University of Agriculture and Technology, \\ Pantnagar, Uttarakhand, India
}

Correspondence should be addressed to Khilap Singh; singh.khilap8@gmail.com

Received 14 December 2015; Accepted 25 October 2016

Academic Editor: Jean-Pierre Corriou

Copyright (C) 2016 K. Singh and M. Kumar. This is an open access article distributed under the Creative Commons Attribution License, which permits unrestricted use, distribution, and reproduction in any medium, provided the original work is properly cited.

\begin{abstract}
A numerical model is developed to examine the effects of thermal radiation on unsteady mixed convection flow of a viscous dissipating incompressible micropolar fluid adjacent to a heated vertical stretching surface in the presence of the buoyancy force and heat generation/absorption. The Rosseland approximation is used to describe the radiative heat flux in the energy equation. The model contains nonlinear coupled partial differential equations which have been converted into ordinary differential equation by using the similarity transformations. The dimensionless governing equations for this investigation are solved by Runge-KuttaFehlberg fourth fifth-order method with shooting technique. Numerical solutions are then obtained and investigated in detail for different interesting parameters such as the local skin-friction coefficient, wall couple stress, and Nusselt number as well as other parametric values such as the velocity, angular velocity, and temperature.
\end{abstract}

\section{Introduction}

The micro polar fluids are those which contain microconstituents that can undergo rotation, the presence of which can affect the hydrodynamics of the flow. The classical Navier-Stokes theory does not describe the flow properties of micropolar fluids, for example, colloidal suspension, polymeric fluids, liquid crystals, fluids with additives, suspension solutions, animal's blood, human blood, body fluids, biofluids, and fluids containing certain additives. Eringen [1] describes the theory of micropolar fluids, which show microrotation effects as well as microinertia. The theory of thermomicropolar fluids was developed by Eringen [2] by extending his theory of micropolar fluids. A good list of references for micropolar fluids is available in Łukaszewicz [3]. Many researchers [4-13] have studied the micropolar fluid flow for different fluid properties over different geometries.

Unsteady mixed convection flow plays an important role in chemical engineering, turbomachinery, aerospace technology, geophysics, and so forth; Zueco et al. [14] studied the unsteady free convection flow of an MHD micropolar fluid through two parallel infinite porous vertical plates. Unsteady mixed convection flow of a micropolar fluid adjacent to a heated vertical surface along with viscous dissipation and the buoyancy force is analyzed by Abd El-Aziz [15]. Hussainn et al. [16] reported the radiation effects on the unsteady boundary layer flow of a micropolar fluid over a stretching permeable sheet. Oahimire and Olajuwon [17] studied the heat and mass transfer effects on an unsteady flow of a chemically reacting micropolar fluid over an infinite vertical porous plate. Rashad [18] studied the unsteady MHD boundary-layer flow and heat transfer for an electrically conducting rotating fluid due to a stretching surface in porous medium in the presence of thermal radiation. Abd El-Aziz [19] investigated the effects of variable viscosity on mixed convection flow along a semi-infinite unsteady stretching sheet with viscous dissipation.

The heat transfer in the fluid flow due to a stretching sheet has attracted considerable attention during the last few decades due to its various applications in many industrial 
and engineering processes such as hot rolling, wire drawing, glass-fiber and paper production, drawing of plastic films, metal and polymer extrusion, and metal spinning. The pioneering work in this area was first made by Crane [20]; he studied boundary layer flow from a linearly stretching plate. Bhargava et al. [21] obtained a finite element solution for the mixed convection micropolar fluid flow in porous stretching sheet with suction. Eldabe and Ouaf [22] examined the heat and mass transfer flow of a micropolar fluid past a stretching surface with Ohmic heating and viscous dissipation. Pal et al. [23] analyzed the effects of thermal radiation and viscous dissipation on mixed convection flow of nanofluid over a nonlinear stretching/shrinking sheet. Singh and Kumar [24] studied boundary layer stagnation point flow of micropolar fluid towards a stretching/shrinking sheet in the presence of melting heat. Turkyilmazoglu [25] analyzed the micropolar fluid flow and heat transfer due to a porous stretching sheet.

Influence of thermal radiation on flow and heat transfer study is much more important in different industries. The heat transfer and temperature profile of a fluid flow over different geometries can be affected significantly at high temperature. Mohamed and Abo-Dahab [26] studied thermal radiation effect on hydromagnetic free convection heat and mass transfer flow of a micropolar fluid over vertical porous plate. The micropolar fluid flow and heat transfer from a porous shrinking sheet were investigated by Bhattacharyya et al. [27]. Prakash and Muthtamilselvan [28] analyzed the effect of thermal radiation on fully developed flow of MHD micropolar fluid through two infinite parallel porous vertical plates.

The effect of heat generation on heat transfer is an important issue in view of various physical problems. Ziabakhsh et al. [29] proposed micropolar fluid flow with heat generation. Bakr [30] investigated the effects of heat source on heat and mass transfer flow of a micropolar fluid in a rotating frame of reference. The heat generation/absorption effects on MHD flow of micropolar fluid through a stretching surface have been studied by Mahmoud and Waheed [31]. Abbasi et al. [32] investigated the Maxwell nanofluid fluid flow and heat transfer in the presence of heat generation/absorption. Mliki et al. [33] examined the influence of Brownian motion and heat generation/absorption over linear/sinusoidally heated cavity. Elgazery [34] analyzed the chemical reaction effect on MHD flow in the presence of temperature dependent viscosity and thermal diffusivity.

The purpose of the present work is to study the effects of thermal radiation on mixed convection flow of a micropolar fluid through an unsteady stretching surface with viscous dissipation and heat generation/absorption. This problem is important in the processing of chemical engineering fluids including polymeric suspensions, lubricant manufacture, and so forth. The nonlinearity of basic equations associated with their inherent mathematical difficulties has led us to use numerical method. Thus the transformed dimensionless pertinent equations are solved numerically by using the Runge-Kutta-Fehlberg fourth fifth-order method along with shooting technique. The velocity, angular velocity, and temperature profiles are shown and the influences of the micropolar parameter, the thermal radiation parameter, the

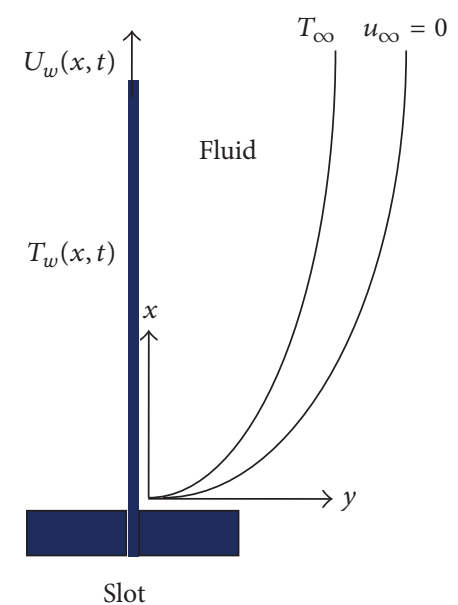

FIgURe 1: Physical model and coordinate system.

unsteadiness parameter, and the buoyancy parameter on the flow and heat transfer characteristics are discussed in detail. To the best of author's knowledge such a study does not appear in the scientific literature.

\section{Mathematical Formulation}

We consider a two-dimensional unsteady mixed convection boundary layer flow of a viscous incompressible micropolar fluid over an elastic, vertical, and impermeable stretching sheet which emerges vertically in the upward direction from a narrow slot with velocity:

$$
U_{w}(x, t)=\frac{a x}{1-\alpha t}
$$

where both $a$ and $\alpha$ are positive constants with dimension per time. The problem is in the presence of thermal radiation and heat generation/absorption. The flow configuration of this problem is illustrated in Figure 1. The positive $x$ coordinate is measured along the stretching sheet with the slot as the origin and the positive $y$ coordinate is measured normal to the sheet in the outward direction towards the fluid. The surface temperature $T_{w}$ of the stretching sheet varies with the distance $x$ from the slot and time $t$ as

$$
T_{w}(x, t)=T_{\infty}+\frac{b x}{(1-\alpha t)^{2}},
$$

where $b$ is a constant with dimension temperature over length and $T_{\infty}$ is the temperature of the ambient fluid. The expressions for $U_{w}(x, t)$ and $T_{w}(x, t)$ in (1) and (2) are valid only for time $t<\alpha^{-1}$ unless $\alpha=0$. Further, expression (1) for velocity of the sheet $U_{w}(x, t)$ reveals that the elastic surface which is fixed at the origin is stretched by applying force in the positive $x$-axis and the effective stretching rate $a /(1-\alpha t)$ increases with time. With the same analogy expression (2) for the surface temperature $T_{w}(x, t)$ depicts a situation in which the surface temperature increases (or decreases) if $b$ is positive (or negative) from $T_{\infty}$ at the slot in proportion to $x$ and such that the amount of temperature increase (or 
decrease) along the sheet increases with time. It is further assumed that the fluid properties are taken to be constant except for the density variation with the temperature in the buoyancy term. Under these assumptions, the governing equations of boundary layer are given in the following form:

$$
\begin{aligned}
& \frac{\partial u}{\partial x}+\frac{\partial v}{\partial y}=0 \\
& \frac{\partial u}{\partial t}+u \frac{\partial u}{\partial x}+v \frac{\partial u}{\partial y} \\
& =\left(\frac{\mu+\kappa}{\rho}\right) \frac{\partial^{2} u}{\partial y^{2}}+\frac{\kappa}{\rho} \frac{\partial N}{\partial x}+g^{*} \beta\left(T-T_{\infty}\right), \\
& \rho j\left(\frac{\partial N}{\partial t}+u \frac{\partial N}{\partial x}+v \frac{\partial N}{\partial y}\right)=\gamma \frac{\partial^{2} N}{\partial y^{2}}-\kappa\left(2 N+\frac{\partial u}{\partial y}\right) \\
& \frac{\partial T}{\partial t}+u \frac{\partial T}{\partial x}+v \frac{\partial T}{\partial y} \\
& =\frac{k}{\rho C_{p}} \frac{\partial^{2} u}{\partial y^{2}}+\left(\frac{\mu+\kappa}{\rho C_{p}}\right)\left(\frac{\partial u}{\partial y}\right)^{2}-\frac{1}{\rho C_{p}} \frac{\partial q_{r}}{\partial y} \\
& \quad+\frac{\theta_{0}}{\rho C_{p}}\left(T-T_{\infty}\right),
\end{aligned}
$$

where $u$ and $v$ are the components of velocity along $x$ and $y$ directions, respectively. Further $\mu$ is dynamic viscosity, $\kappa$ is vortex viscosity, $\rho$ is fluid density, $g^{*}$ is the gravitational acceleration, $\beta$ is the volumetric coefficient of the thermal expansion, $v$ is kinematic viscosity, $j$ is microinertia density, $\gamma$ is the spin-gradient viscosity, $N$ is the component of microrotation whose direction of rotation lies in the $x-y$ plane, $T$ is the temperature, $k$ is the thermal conductivity of the fluid, $C_{p}$ is the heat capacity at constant pressure $p, q_{r}$ is the radiative heat flux, $\theta_{0}$ is the heat generation/absorption coefficient, and $T_{\infty}$ is the temperature of the ambient fluid.

The appropriate boundary conditions for the problem are

$$
\begin{aligned}
& u=U_{w}(x, t), \\
& v=0, \\
& N=0, \\
& T=T_{w}(x, t) \\
& u \rightarrow 0, \\
& N \longrightarrow 0, \\
& T \longrightarrow T_{\infty}
\end{aligned}
$$

as $y \longrightarrow \infty$.

Using Roseland's approximation, the radiative heat flux $q_{r}$ is modeled as

$$
q_{r}=-\left(\frac{4 \sigma}{3 k_{1}}\right) \frac{\partial T^{4}}{\partial y}
$$

where $\sigma$ is the Stefan-Boltzmann constant and $k_{1}$ is the absorption coefficient. Assuming that the difference in temperature within the flow is such that $T^{4}$ can be expressed as a linear combination of the temperature, we expand $T^{4}$ in Taylor's series about $T_{\infty}$ as follows:

$$
T^{4}=T_{\infty}^{4}+4 T_{\infty}^{3}\left(T-T_{\infty}\right)+6 T_{\infty}^{2}\left(T-T_{\infty}\right)^{2}+\cdots
$$

and neglecting higher order terms beyond the first degree in $\left(T-T_{\infty}\right)$, we have

$$
T^{4} \approx 3 T_{\infty}^{4}+4 T_{\infty}^{3} T
$$

Differentiating (8) with respect to $y$ and using (10) we get

$$
\frac{\partial q_{r}}{\partial y}=-\frac{16 T_{\infty}^{3} \sigma}{3 k_{1}} \frac{\partial^{2} T}{\partial y^{2}} .
$$

Using (11) in (6) we obtain

$$
\begin{aligned}
\frac{\partial T}{\partial t}+u \frac{\partial T}{\partial x}+v \frac{\partial T}{\partial y}= & \frac{k}{\rho C_{p}} \frac{\partial^{2} u}{\partial y^{2}}+\frac{16 \sigma T_{\infty}^{3}}{3 k_{1} \rho C_{p}} \frac{\partial^{2} T}{\partial y^{2}} \\
& +\left(\frac{\mu+\kappa}{\rho C_{p}}\right)\left(\frac{\partial u}{\partial y}\right)^{2} \\
& +\frac{\theta_{0}}{\rho C_{p}}\left(T-T_{\infty}\right) .
\end{aligned}
$$

The continuity equation (3) is satisfied by introducing the stream function $\psi$ such that

$$
\begin{aligned}
& u=\frac{\partial \psi}{\partial y}=U_{w} f^{\prime}(\eta) \\
& v=-\frac{\partial \psi}{\partial x}=-\sqrt{\frac{2 v}{1-\alpha t}} f(\eta) .
\end{aligned}
$$

Equations (4), (5), and (12) can be transformed into a set of nonlinear ordinary differential equations by using the following similarity transformations:

$$
\begin{aligned}
& \eta=\sqrt{\frac{a}{v(1-\alpha t)} y,} \\
& \psi=\sqrt{\frac{v a}{1-\alpha t} x f(\eta),} \\
& N=\sqrt{\frac{a^{3}}{v(1-\alpha t)^{3}} x g(\eta),} \\
& T=T_{\infty}+\frac{b x}{(1-\alpha t)^{2}} \theta(\eta) .
\end{aligned}
$$

The transformed ordinary differential equations are

$$
\begin{aligned}
& (1+K) f^{\prime \prime \prime}+f f^{\prime \prime}-\left(f^{\prime}\right)^{2}-\frac{A}{2}\left(2 f^{\prime}+\eta f^{\prime \prime}\right)+K g^{\prime} \\
& \quad+\xi \theta=0 \\
& \lambda_{0} g^{\prime \prime}(\eta)+f g^{\prime}-f^{\prime} h-\frac{A}{2}\left(3 g+\eta g^{\prime}\right)-K B(2 g \\
& \left.\quad+f^{\prime \prime}\right)=0
\end{aligned}
$$


TABLE 1: Comparison of $-\theta^{\prime}(0)$ for different values of $A$ and $\xi$ when $K=\mathrm{Ec}=R=0$ and $\operatorname{Pr}=1$.

\begin{tabular}{llcc}
\hline$A$ & $\xi$ & Abd El-Aziz [15] & Present results \\
\hline 0 & 0 & 1.00000000 & 1.00000000 \\
0 & 1 & 1.08727816 & 1.08727818 \\
0 & 2 & 1.14233927 & 1.14233928 \\
0 & 3 & 1.18529030 & 1.18529032 \\
1 & 0 & 1.68199254 & 1.68199255 \\
1 & 1 & 1.70391279 & 1.70391281 \\
\hline
\end{tabular}

$$
\begin{aligned}
& (3 R+4) \theta^{\prime \prime}+3 R \operatorname{Pr}\left[f \theta^{\prime}-f^{\prime} g-\frac{A}{2}\left(4 \theta+\eta \theta^{\prime}\right)\right. \\
& \left.+\operatorname{Ec}(1+K)\left(f^{\prime \prime}\right)^{2}+H \theta\right]=0 .
\end{aligned}
$$

The boundary conditions (7) reduce to

$$
\begin{aligned}
f(\eta) & =0, \\
f^{\prime}(\eta) & =1, \\
g(\eta) & =0, \\
\theta(\eta) & =1 \\
& \text { at } \eta=0, \\
f^{\prime}(\eta) & =0, \\
g(\eta) & =0, \\
\theta(\eta) & =0 \quad \text { as } \eta=\infty
\end{aligned}
$$

where prime denotes ordinary differentiation with respect to $\eta, K=\kappa / \rho$ is the micropolar parameter, $A=\alpha / a$ is the unsteadiness parameter, $\theta=T-T_{\infty} / T_{w}-T_{\infty}$ is the nondimensional temperature, $\xi=g \beta b / a^{2}$ is the mixed convection or buoyancy parameter, $\lambda_{0}=\gamma / \mu j$ is the spingradient viscosity parameter, $B=v(1-\alpha t) / j b=v x / j U_{w}$ is the microinertia density parameter, $\operatorname{Pr}=v / \alpha$ is the Prandtl number, $R=k k_{1} / 4 \sigma T_{\infty}^{3}$ is the thermal radiation parameter, Ec $=U_{w}^{2} / C_{p}\left(T_{w}-T_{\infty}\right)$ is the Eckert number, and $H=\theta_{0} x / \rho C_{p} U_{w}$ is the local heat generation/absorption parameter.

It is worth mentioning that, for $\xi>0\left(T_{w}>T_{\infty}\right)$, buoyancy forces act in the direction of the mainstream and fluid is accelerated in the manner of a favorable pressure gradient (assisting flow). When $\xi<0\left(T_{w}<T_{\infty}\right)$, buoyancy forces oppose the stretching induced flow, retarding the fluid in the boundary layer, acting as an adverse pressure gradient (opposing flow). Further, according to the definition of the viscous dissipation parameter, $\mathrm{Ec}<0$ in the case of assisting flow $(\xi>0)$ and $\mathrm{Ec}<0$ in the case of opposing flow $(\xi<0)$.

The most important physical quantities for the problem are the local skin-friction coefficient $C_{f x}$, the local wall couple stress coefficient $M_{w x}$, and the local Nusselt number $\mathrm{Nu}_{x}$ which are defined by

$$
\begin{aligned}
C_{f x} & =\frac{2}{\rho U_{w}^{2}}\left[(\mu+\kappa)\left(\frac{\partial u}{\partial y}\right)_{y=0}\right]=2(1+K) f^{\prime \prime}(0), \\
M_{w x} & =\gamma\left(\frac{\partial N}{\partial y}\right)_{y=0}=\frac{\gamma a U_{w}}{v(1-\alpha t)} g^{\prime}(0), \\
\mathrm{Nu}_{x} & =-\frac{x}{T_{w}-T_{\infty}}\left(\frac{\partial T}{\partial y}\right)_{y=0}=-\operatorname{Re}_{x}^{1 / 2} \theta^{\prime}(0),
\end{aligned}
$$

where $\operatorname{Re}_{x}=x U_{w} / v$ is the local Reynolds number.

\section{Method of Solution}

The nonlinear differential equations (15) subject to boundary conditions (16) have been solved by using Runge-KuttaFehlberg fourth fifth-order method along with the shooting technique. This method is based on the discretization of the problem domain and the calculation of unknown boundary conditions.

The domain of the problem is discretized and the boundary conditions for $\eta=\infty$ are replaced by $f^{\prime}\left(\eta_{\max }\right)=0$, $g\left(\eta_{\max }\right)=0$, and $\theta\left(\eta_{\max }\right)=0$, where $\eta_{\infty}$ is a sufficiently large value of $\eta$ at which boundary conditions (16) are satisfied. The authors ran the computer code written in MATLAB for different values of $\eta_{\max }$ and step size $\Delta \eta$. They have seen that that there is no or negligible change in the velocity, angular velocity, and temperature for values of $\eta$ greater than 7. Therefore in the present paper we have set $\eta_{\infty}=7$ and step size $\Delta \eta=0.001$. To solve the problem the nonlinear equations (15) have been first converted into seven firstorder linear ordinary differential equations. There are four initial conditions at $\eta=0$ and three boundary conditions at $\eta=\infty$. To find the solution of the problem, one will need three more conditions at $\eta=0$, that is, the values of $f^{\prime \prime}(0), g^{\prime}(0)$, and $\theta^{\prime}(0)$. These conditions have been found by the shooting technique. Finally the problem has been solved by the Runge-Kutta-Fehlberg method along with calculated boundary conditions.

\section{Results and Discussion}

In order to validate the numerical results obtained, we compare our results with those reported by Abd El-Aziz [11] as shown in Table 1, and they are found to be in a favorable agreement. In the simulation the default values of 


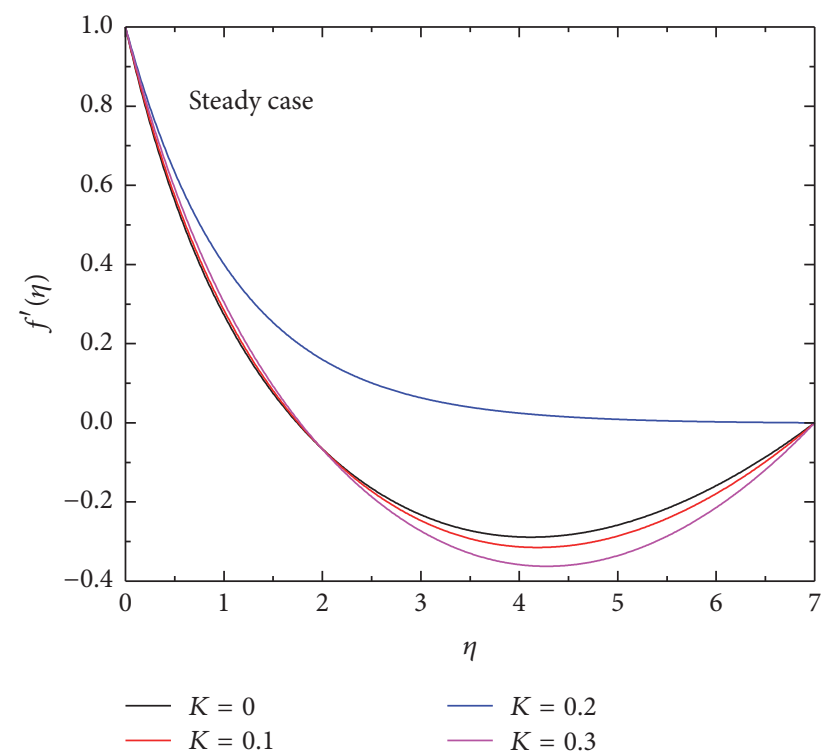

FIGURE 2: Velocity profiles for various values of $K$ when $A=0, R=$ 0.5 , and $\xi=0$.

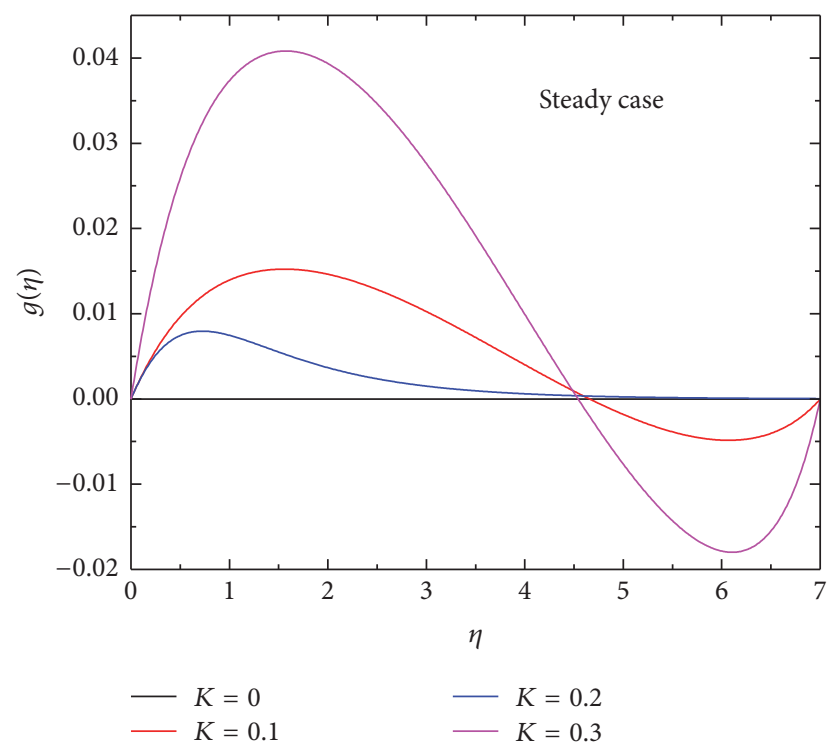

FIGURE 3: Microrotation profiles for various values of $K$ when $A=0$, $R=0.5$, and $\xi=0$.

the parameters are considered as $\lambda_{0}=0.3, B=0.1, \operatorname{Pr}=0.72$, $\mathrm{Ec}=0.05$, and $H=0$ unless otherwise specified.

Figures 2-4 show the effects of micropolar parameter $K$ on the velocity $f^{\prime}(\eta)$, microrotation $g(\eta)$, and temperature $\theta(\eta)$ profiles in the absence of unsteadiness $(A=0)$ when $R=$ 0.5 and $\xi=0$. Figure 2 depicts that, with an increase in values of $K$, the velocity increases slightly near the surface and after a certain distance it decreases, but at $K=2$ it increases rapidly. It is clear from Figure 3 that the microrotation $g(\eta)$ increases with increase in $K$, while at $K=0.2$ it decreases quickly. Further we notice from this figure that the microrotation takes negative values for large values of $K(0.1$ and 0.3$)$.

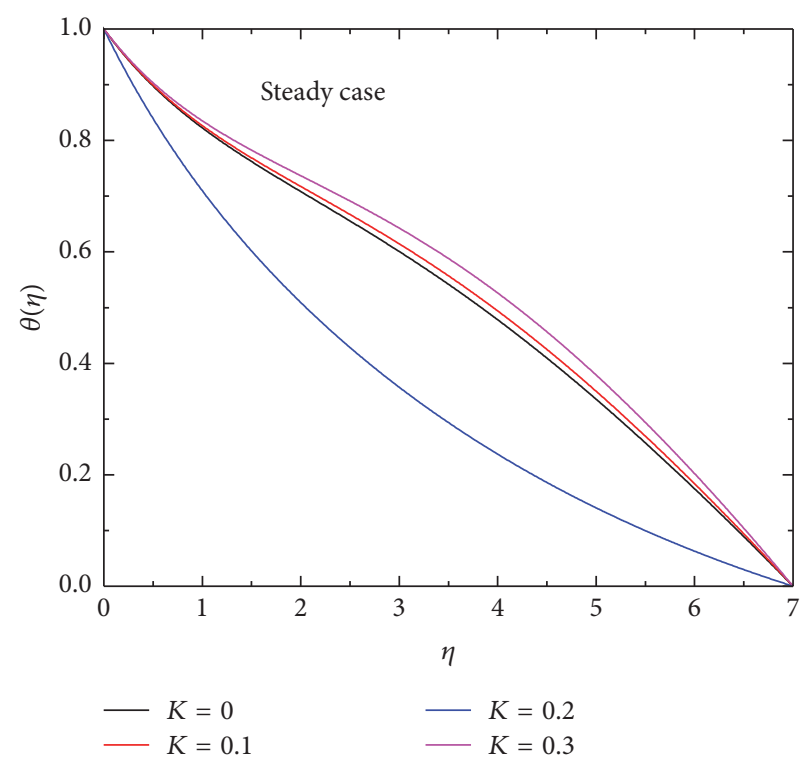

FIgURE 4: Temperature profiles for various values of $K$ when $A=0$, $R=0.5$, and $\xi=0$.

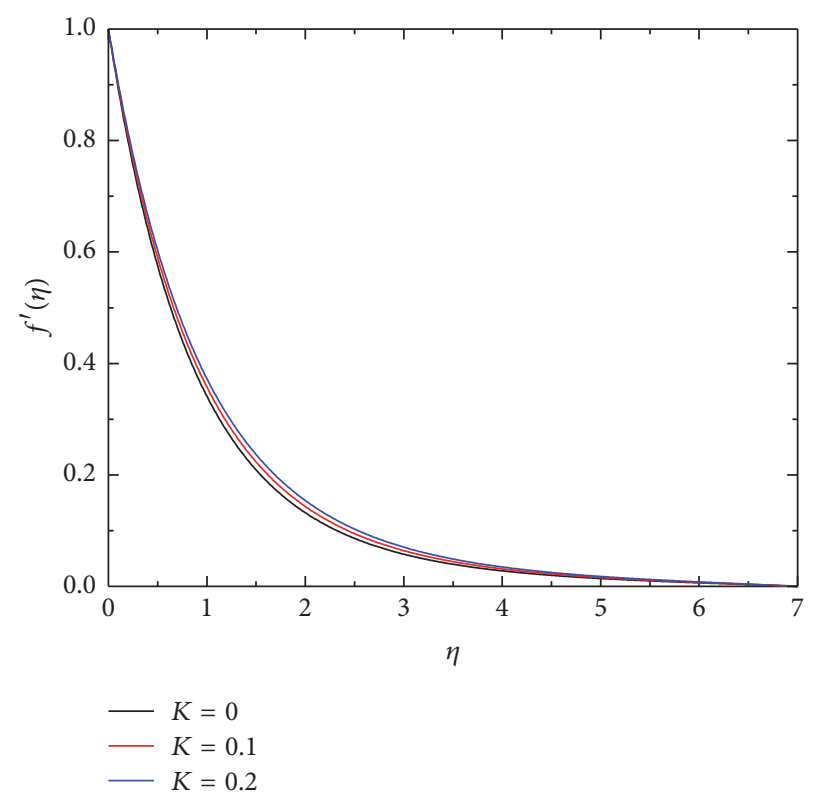

FIgURE 5: Velocity profiles for various values of $K$ when $A=0.4$, $R=0.5$, and $\xi=0$.

Physically it means that there is a reversed flow for this case. The fluid temperature increases with an increase in the values of $K$, and however at $K=0.2$ it decreases rapidly as shown in Figure 4.

Figures 5-7 depict the variation of the velocity $f^{\prime}(\eta)$, microrotation $g(\eta)$, and temperature $\theta(\eta)$ profiles for different values of the micropolar parameter $K$ when $A=0.4$, $R=0.5$, and $\xi=0$. It is clear from Figure 5 that velocity $f^{\prime}(\eta)$ increases with increasing value of $K$. It is noticed from Figure 6 that an increase in the values of micropolar parameter $K$ leads to rise in the values of the microrotation 


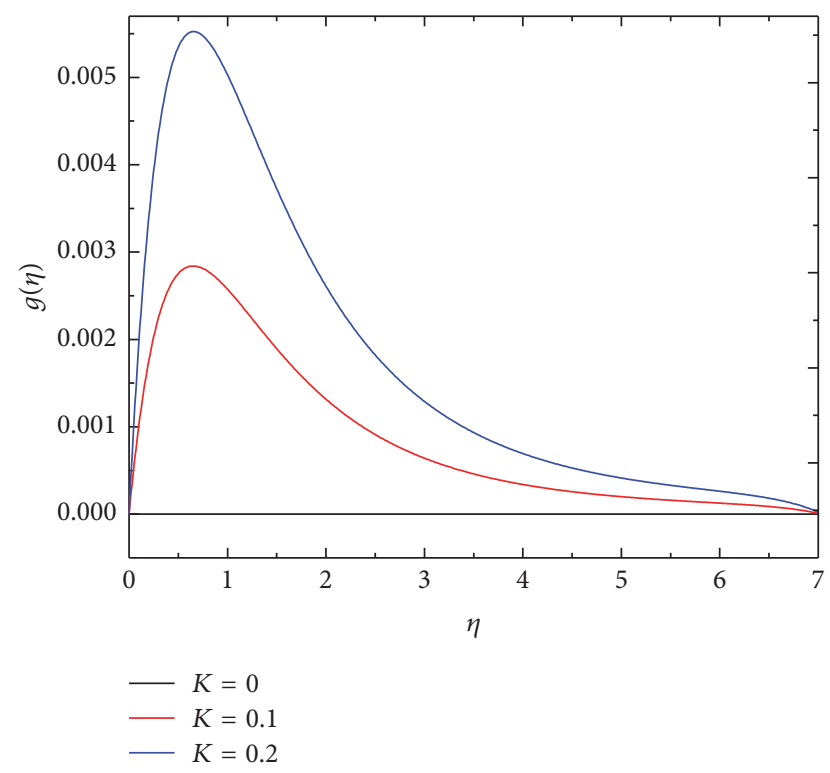

FIgURE 6: Microrotation profiles for various values of $K$ when $A=$ $0.4, R=0.5$, and $\xi=0$.

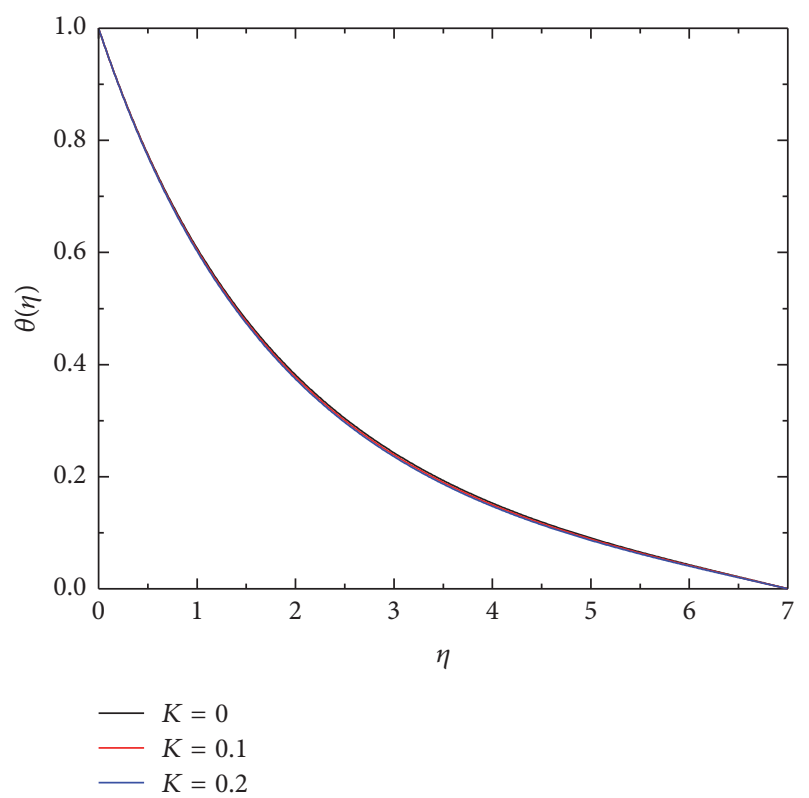

Figure 7: Temperature profiles for various values of $K$ when $A=0$, $R=0.5$, and $\xi=0$.

$g(\eta)$, also it can be seen that peak point of this profile shifts to the surface by increasing values of $K(0.1$ and 0.2$)$, while for $K=0$ microrotation $g(\eta)$ has constant value zero. It is evident from Figure 7 that the temperature decreases with the increasing values of micropolar parameter $K$.

Figure 8 presents the effects of the thermal radiation parameter $R$ on temperature profile $\theta(\eta)$ when $K=0.2, A=$ 0 , and $\xi=0$. It is clear from this figure that the temperature profile reduces with increasing values of the thermal radiation parameter $R$.

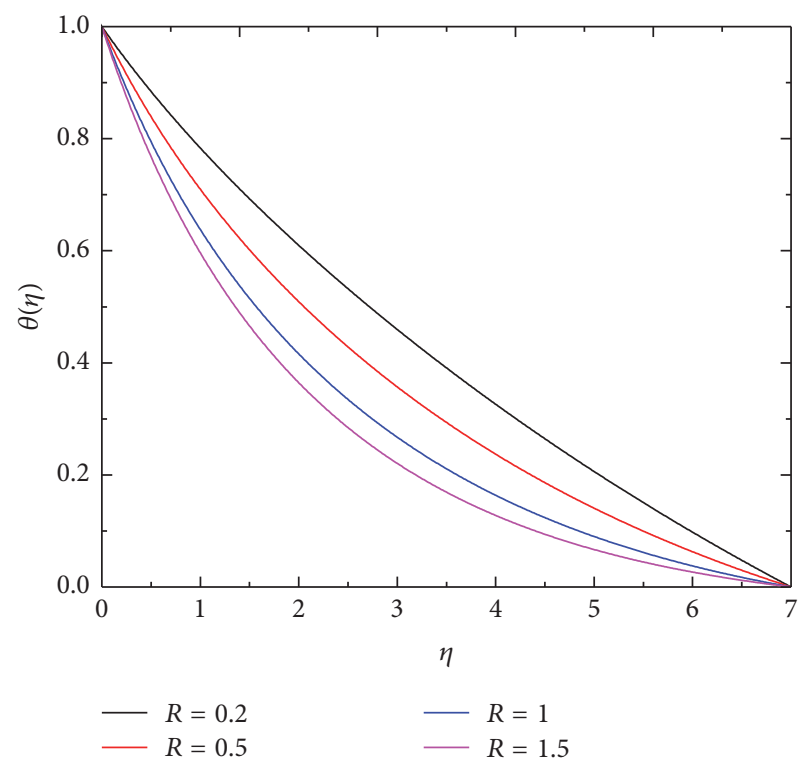

FIGURE 8: Temperature profiles for various values of $R$ when $K=0.2$, $A=0$, and $\xi=0$.

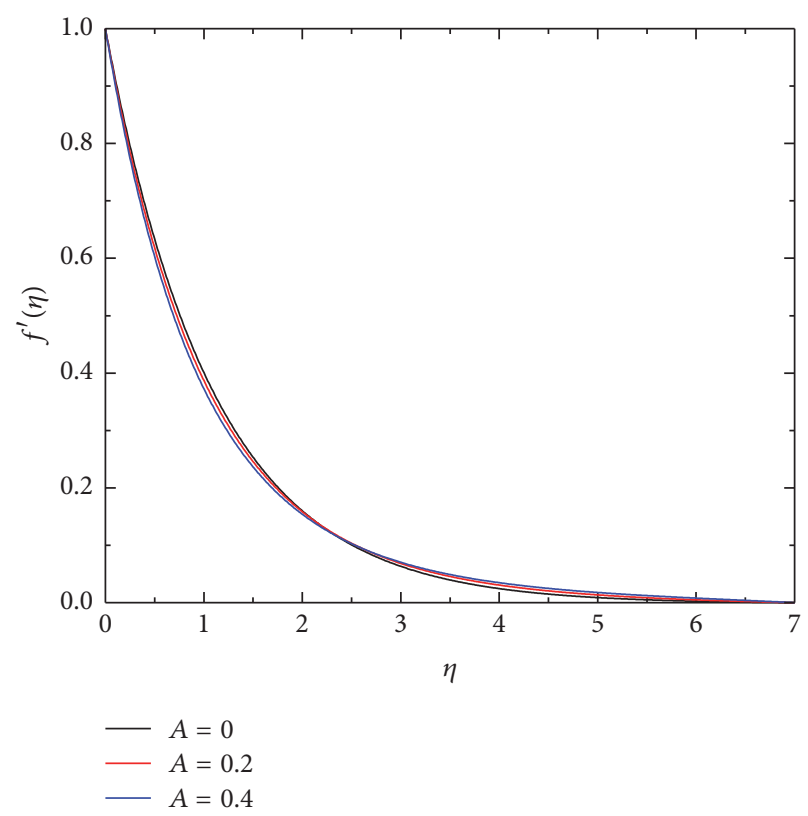

FIgURE 9: Velocity profiles for various values of $A$ when $K=0.2$, $R=0.5$, and $\xi=0$.

Figures 9-11 exhibit the velocity, microrotation, and temperature profiles for various values of unsteadiness parameter $A$ when $K=0.2, R=0.5$, and $\xi=0$. It is noticed from Figure 9 that, with an increase in unsteadiness parameter, the velocity $f^{\prime}(\eta)$ decreases and after a certain distance away from the surface it increases. It is seen from Figure 10 that the microrotation $g(\eta)$ is initially smaller, in particular when $\eta$ is less than approximately 4 and then $g(\eta)$ increases slightly for larger $A$. Furthermore, the microrotation increases to a maximum point near the surface from which it starts to decrease and reaches zero near the free stream with the 


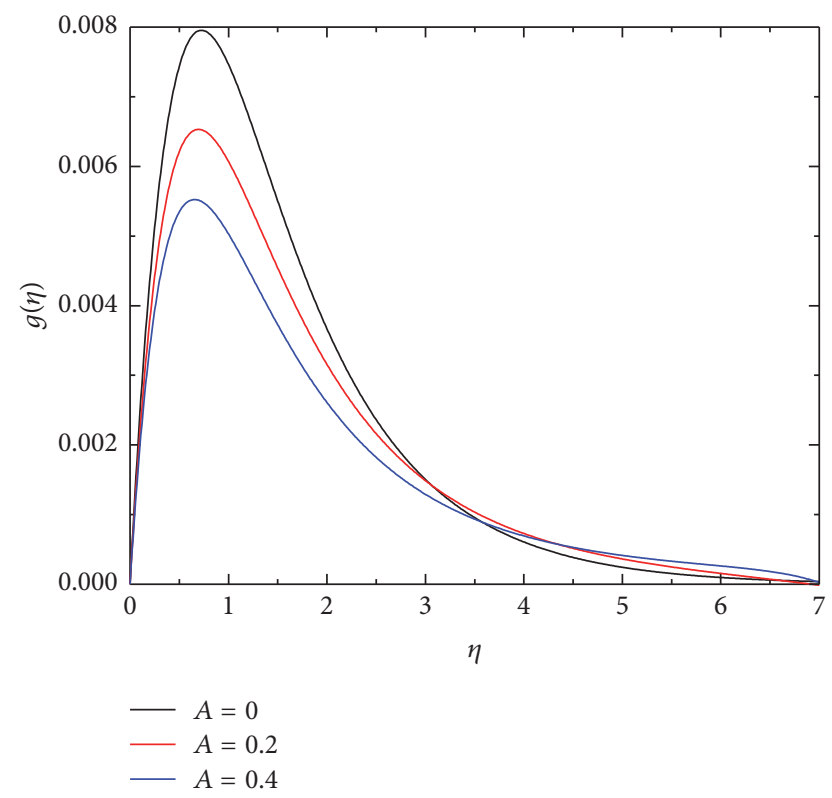

FIGURE 10: Microrotation profiles for various values of $A$ when $K=$ $0.2, R=0.5$, and $\xi=0$.

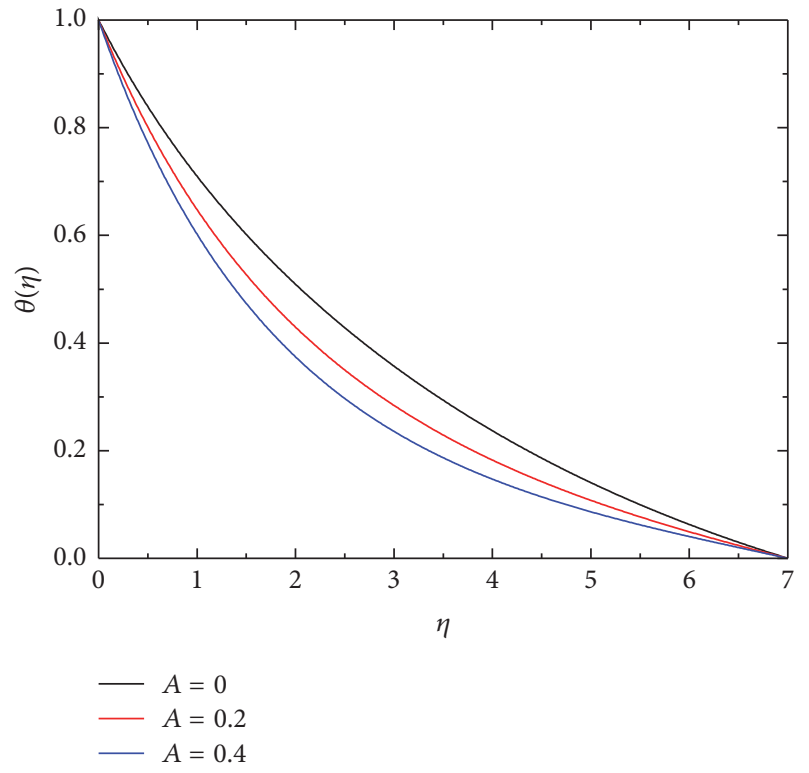

FIGURE 11: Temperature profiles for various values of $A$ when $K=$ $0.2, R=0.5$, and $\xi=0$.

increasing values of $\eta$. It is clear from Figure 11 that the temperature reduces monotonically with an increase in the values of $A$.

Figures 12-14 depict the effects of buoyancy parameter $\xi$ on the velocity $f^{\prime}(\eta)$, microrotation $g(\eta)$, and temperature $\theta(\eta)$ profiles when $A=0.2, R=0.5$, and $K=0.2$. For assisting flow $\xi>0$, it is seen from Figure 12 that velocity increases with $\xi$; that is, increasing of $\xi$ has tendency to induce more flow in the boundary layer. For the opposing flows $(\xi<0)$, on the other hand the effect of buoyancy is to reduce the velocity compared to those for pure forced convection $(\xi=0)$. This

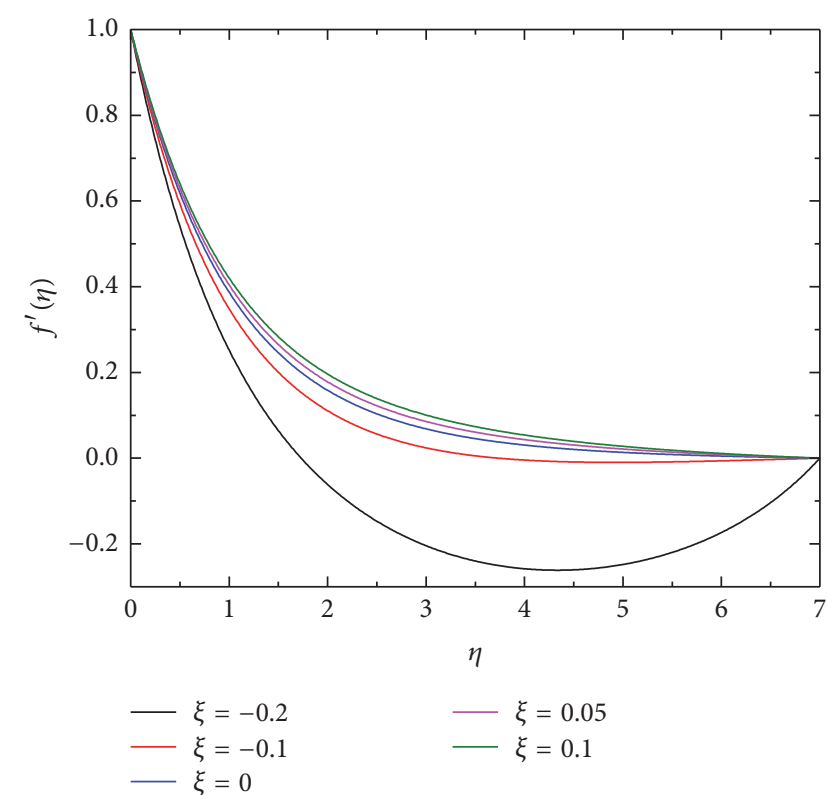

FIGURE 12: Velocity profiles for various values of $\xi$ when $A=0.2$, $R=0.5$, and $K=0.2$.

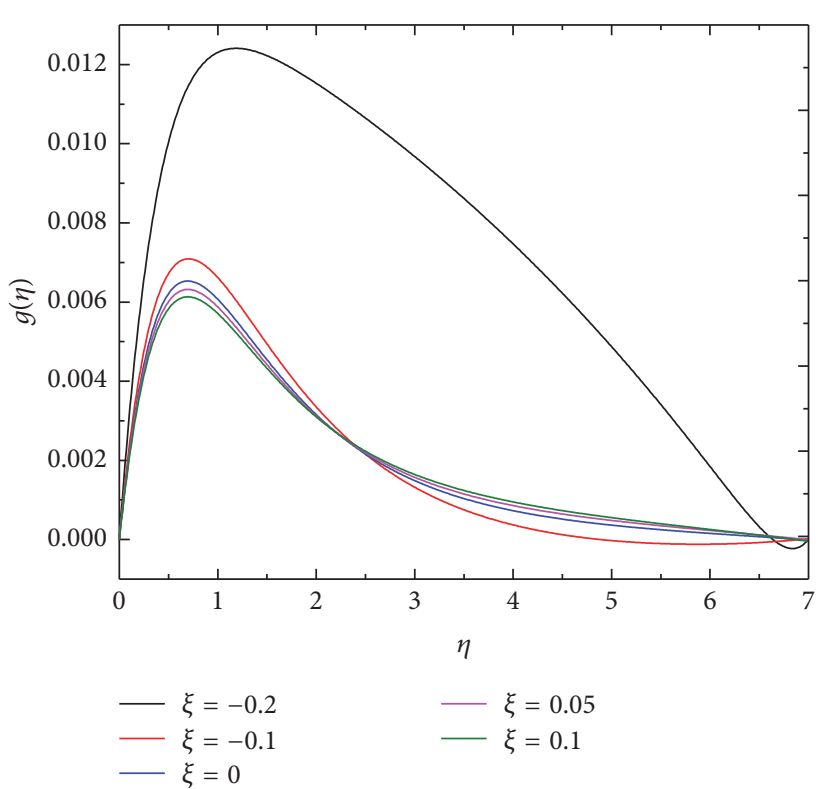

FIGURE 13: Microrotation profiles for various values of $\xi$ when $A=$ $0.2, R=0.5$, and $K=0.2$.

is because a positive $\xi$ induces favorable pressure gradient that enhances the flow in boundary layer, while a negative $\xi$ produces an adverse pressure gradient that slows down the fluid flow. Also for $\xi=-0.2$ the dimensionless fluid velocity $f^{\prime}(\eta)$ decreases rapidly with $\eta$ first, arriving at a negative minimum value, and then increases to its free surface value. It is noted from Figure 13 that the angular velocity $g(\eta)$ first decreases with $\xi$ near the sheet surface where $0 \leq \eta<\eta_{0} \cong 2.4$ and increases with $\xi$ for $\eta>\eta_{0}$. Also it is seen from this figure that for $\xi=-2$ the angular velocity $g(\eta)$ increases greatly 
TABLE 2: Computed values of $f^{\prime \prime}(0), g^{\prime}(0)$, and $-\theta^{\prime}(0)$ for various values of $A, \xi, K$, and $R$.

\begin{tabular}{lcccccc}
\hline$A$ & $\xi$ & $K$ & $R$ & $f^{\prime \prime}(0)$ & $g^{\prime}(0)$ & $-\theta^{\prime}(0)$ \\
\hline 0 & 0 & 0.2 & 0.5 & -0.91248 & 0.027431 & 0.36217 \\
0.2 & 0 & 0.2 & 0.5 & -0.97460 & 0.024750 & 0.45577 \\
0.4 & 0 & 0.2 & 0.5 & -1.03540 & 0.022970 & 0.53131 \\
0.2 & -0.2 & 0.2 & 0.5 & -1.16166 & 0.034717 & 0.38816 \\
0.2 & -0.1 & 0.2 & 0.5 & -1.04170 & 0.026600 & 0.44245 \\
0.2 & 0.05 & 0.2 & 0.5 & -0.94316 & 0.023959 & 0.46128 \\
0.2 & 0.1 & 0.2 & 0.5 & -0.91260 & 0.023225 & 0 \\
0 & 0 & 0 & 0.5 & -1.08705 & 0.026701 & 0.243760 \\
0 & 0 & 0.1 & 0.5 & -1.04570 & 0.072558 & 0.240340 \\
0 & 0 & 0.3 & 0.5 & -0.97566 & 0.027431 & 0.232590 \\
0 & 0 & 0.2 & 0.2 & -0.91248 & 0.027431 & 0.251050 \\
0 & 0 & 0.2 & 1 & -0.91248 & & 0.475040 \\
\hline
\end{tabular}

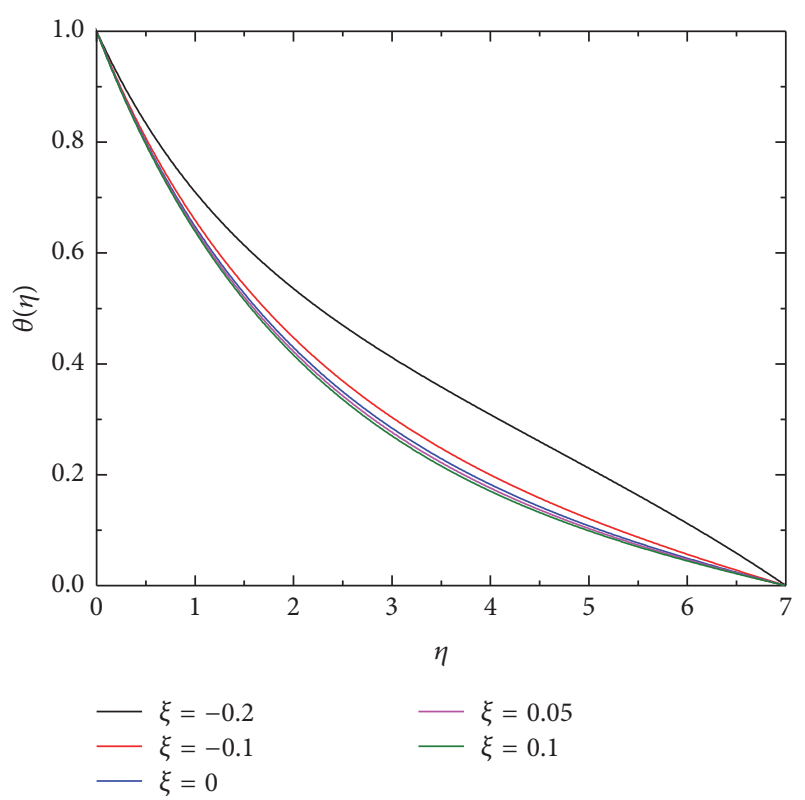

FIgURE 14: Temperature profiles for various values of $\xi$ when $A=$ $0.2, R=0.5$, and $K=0.2$.

near the sheet surface where $0 \leq \eta<\eta_{0} \cong 1.25$, whereas for $\eta>\eta_{0}$ it decreases. Figure 14 shows that the effects of buoyancy parameter $\xi$ are to decrease the temperature $\theta(\eta)$ in the case of assisting flow $\xi>0$ and increase it in the case of opposing flow $\xi<0$.

The effects of the unsteadiness parameter $A$, the buoyancy parameter $\xi$, the micropolar parameter $K$, and the thermal radiation parameter $R$ on local skin-friction coefficient $f^{\prime \prime}(0)$, local wall couple stress $g^{\prime}(0)$, and local Nusselt number $-\theta^{\prime}(0)$ are given in Table 2. It is clear from Table 2 that the local skin-friction coefficient increases with an increase in the values of $K$ and in the case of the assisting flow $\xi>0$, while it decreases with increasing values of $A$ and in the case of opposing flow $\xi<0$. Further, it is seen from Table 2 that the effect of increasing values of $K$ is to increase local couple stress coefficient, whereas increasing $A$ and $\xi$ decreases local couple stress coefficient and there is no variation in local couple stress coefficient with $R$. In addition, the heat transfer rate increases with an increase in $A, R$ and in the case of the assisting flow $\xi>0$. However, the heat transfer rate decreases with increasing the value of $K$ and in the case of opposing flow $\xi<0$.

\section{Conclusions}

The present work deals with the numerical analysis of thermal radiation effects of a mixed convection flow over an unsteady stretching surface. Fluid is a micropolar fluid in the presence of viscous dissipation and heat generation/absorption. The relevant nonlinear partial differential equations were transformed to a set of ordinary differential equations and then are solved numerically using the Runge-Kutta-Fehlberg fourth fifth-order method along with shooting technique. Conclusions drawn from the numerical results are as follows:

(i) Temperature reduces with increase in the values of the unsteadiness parameter, buoyancy parameter, and thermal radiation parameter.

(ii) The skin-friction is enhanced with an increase in the values of the micropolar parameter and buoyancy parameter, while it decreases with the increase of unsteadiness parameter.

(iii) The increasing value of the micropolar parameter is to increase the couple stress, whereas increasing unsteadiness and buoyancy parameter decreases the couple stress.

(iv) The rate of heat transfer increases with the unsteadiness parameter, buoyancy parameter, and thermal radiation parameter; however rate of heat transfer decreases with increasing micropolar parameter and thermal radiation parameter. 


\section{Nomenclature}

$\begin{array}{ll}A: & \text { Unsteadiness parameter } \\ a: & \text { Constant }\left[\mathrm{m}^{-1}\right] \\ B: & \text { Microinertia density parameter } \\ b: & \text { Constant }\left[\mathrm{m}^{-1} \theta\right] \\ C_{f x}: & \text { Coefficient of local skin-friction } \\ C_{p}: & \text { Specific heat at constant pressure }\left[\mathrm{J} \mathrm{kg}^{-1} \mathrm{~K}^{-1}\right] \\ \mathrm{Ec}: & \text { Eckert number } \\ H: & \text { Heat generation/absorption parameter } \\ g^{*}: & \text { Acceleration due to gravity }\left[\mathrm{m} \mathrm{s}^{-2}\right] \\ j: & \text { Microinertia density }\left[\mathrm{m}^{2}\right] \\ K: & \text { Micropolar parameter } \\ k: & \text { Thermal conductivity of the fluid }\left[\mathrm{W} \mathrm{m}^{-1} \mathrm{~K}^{-1}\right] \\ k_{1}: & \text { Absorption coefficient } \\ M_{w x}: & \text { Coefficient of local wall couple stress } \\ N: & \text { Component of microrotation }\left[\mathrm{rad} \mathrm{s}^{-1}\right] \\ \mathrm{Nu} & \text { Nusselt number } \\ \mathrm{Pr}_{x}: & \text { Prandtl number } \\ q_{r}: & \text { Radiative heat flux } \\ R: & \text { Thermal radiation parameter } \\ \mathrm{Re} & \\ T: & \text { Local Reynolds number } \\ u, v: & \text { Temperature }[\mathrm{K}] \\ x, y: & \text { Axial and perpendicular coordinates }\left[\mathrm{m}^{-1} .\right. \\ & \end{array}$

Greek Symbols

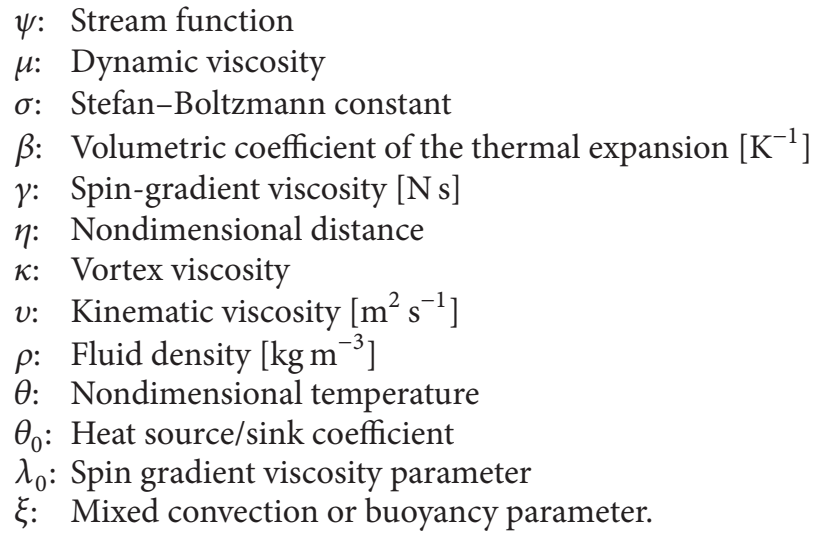

Subscripts

$\infty$ : Free stream condition

$w$ : Condition at the wall of stretching sheet.

\section{Superscript}

': Derivative with respect to $\eta$.

\section{Competing Interests}

The authors declare that there is no conflict of interests regarding the publication of this paper. The authors also confirm that the mentioned received funding in Acknowledgments did not lead to any conflict of interests regarding the publication of this manuscript.

\section{Acknowledgments}

The authors wish to express their very sincere thanks to honorable referees for their valuable comments and suggestions for the improvement of the manuscript. The first author gratefully acknowledges the financial support of UGC, India, under F. 17-97/2008 (SA-I), for pursuing this work.

\section{References}

[1] A. C. Eringen, “Theory of micropolar fluids," Journal of Mathematics and Mechanics, vol. 16, pp. 1-18, 1966.

[2] A. C. Eringen, "Theory of thermomicrofluids," Journal of Mathematical Analysis and Applications, vol. 38, no. 2, pp. 480-496, 1972.

[3] G. Łukaszewicz, Micropolar Fluids: Theory and Application, Birkhäuser, Basel, Switzerland, 1999.

[4] M. W. Heruska, L. T. Watson, and K. K. Sankara, "Micropolar flow past a porous stretching sheet," Computers \& Fluids, vol. 14, no. 2, pp. 117-129, 1986.

[5] M. Ashraf, M. A. Kamal, and K. S. Syed, "Numerical study of asymmetric laminar flow of micropolar fluids in a porous channel," Computers \& Fluids, vol. 38, no. 10, pp. 1895-1902, 2009.

[6] N. A. Yacob, A. Ishak, and I. Pop, "Melting heat transfer in boundary layer stagnation-point flow towards a stretching/ shrinking sheet in a micropolar fluid," Computers and Fluids, vol. 47, no. 1, pp. 16-21, 2011.

[7] Z. Alloui and P. Vasseur, "Double-diffusive and Soret-induced convection in a micropolar fluid layer," Computers \& Fluids, vol. 60, pp. 99-107, 2012.

[8] J. Chen, C. Liang, and J. D. Lee, "Numerical simulation for unsteady compressible Micropolar fluid flow," Computers and Fluids, vol. 66, pp. 1-9, 2012.

[9] K. Singh and M. Kumar, "The effect of chemical reaction and double stratification on MHD free convection in a micropolar fluid with heat generation and Ohmic heating," Jordan Journal of Mechanical and Industrial Engineering, vol. 9, no. 4, pp. 279288, 2015.

[10] F. Mabood, S. M. Ibrahim, M. M. Rashidi, M. S. Shadloo, and G. Lorenzini, "Non-uniform heat source/sink and Soret effects on MHD non-Darcian convective flow past a stretching sheet in a micropolar fluid with radiation," International Journal of Heat and Mass Transfer, vol. 93, pp. 674-682, 2016.

[11] Z. Uddin, M. Kumar, and S. Harmand, "Influence of thermal radiation and heat generation//absorption on MHD heat transfer flow of a micropolar fluid past a wedge with hall and ion slip currents," Thermal Science, vol. 18, no. 2, pp. S489-S502, 2014.

[12] D. Pal and S. Chatterjee, "MHD mixed convection stagnationpoint flow of a micropolar fluid in a porous medium towards a heated stretching sheet with thermal radiation," Mathematical Modelling and Analysis, vol. 17, no. 4, pp. 498-518, 2012.

[13] M. Abd El-Aziz, "Viscous dissipation effect on mixed convection flow of a micropolar fluid over an exponentially stretching sheet," Canadian Journal of Physics, vol. 87, no. 4, pp. 359-368, 2009. 
[14] J. Zueco, P. Eguía, M. Abd El-Aziz, J. Collazo, and D. Patiño, "Unsteady MHD free convection of a micropolar fluid between two parallel porous vertical walls with convection from the ambient," International Communications in Heat and Mass Transfer, vol. 36, no. 3, pp. 203-209, 2009.

[15] M. Abd El-Aziz, "Mixed convection flow of a micropolar fluid from an unsteady stretching surface with viscous dissipation," Journal of the Egyptian Mathematical Society, vol. 21, no. 3, pp. 385-394, 2013.

[16] M. Hussain, M. Ashraf, S. Nadeem, and M. Khan, "Radiation effects on the thermal boundary layer flow of a micropolar fluid towards a permeable stretching sheet," Journal of the Franklin Institute, vol. 350, no. 1, pp. 194-210, 2013.

[17] J. Oahimire and B. Olajuwon, "Effect of Hall current and thermal radiation on heat and mass transfer of a chemically reacting MHD flow of a micropolar fluid through a porous medium," Journal of King Saud University_Engineering Sciences, vol. 26, no. 2, pp. 112-121, 2014.

[18] A. M. Rashad, "Effects of radiation and variable viscosity on unsteady MHD flow of a rotating fluid from stretching surface in porous medium," Journal of the Egyptian Mathematical Society, vol. 22, no. 1, pp. 134-142, 2014.

[19] M. Abd El-Aziz, "Unsteady mixed convection heat transfer along a vertical stretching surface with variable viscosity and viscous dissipation," Journal of the Egyptian Mathematical Society, vol. 22, no. 3, pp. 529-537, 2014.

[20] L. J. Crane, "Flow past a stretching plate," Zeitschrift für angewandte Mathematik und Physik, vol. 21, no. 4, pp. 645-647, 1970.

[21] R. Bhargava, L. Kumar, and H. S. Takhar, "Finite element solution of mixed convection micropolar flow driven by a porous stretching sheet," International Journal of Engineering Science, vol. 41, no. 18, pp. 2161-2178, 2003.

[22] N. T. Eldabe and M. E. M. Ouaf, "Chebyshev finite difference method for heat and mass transfer in a hydromagnetic flow of a micropolar fluid past a stretching surface with Ohmic heating and viscous dissipation," Applied Mathematics and Computation, vol. 177, no. 2, pp. 561-571, 2006.

[23] D. Pal, G. Mandal, and K. Vajravelu, "MHD convection-dissipation heat transfer over a non-linear stretching and shrinking sheets in nanofluids with thermal radiation," International Journal of Heat and Mass Transfer, vol. 65, pp. 481-490, 2013.

[24] K. Singh and M. Kumar, "Melting heat transfer in boundary layer stagnation point flow of MHD micro - polar fluid towards a stretching/shrinking surface," Jordan Journal of Mechanical and Industrial Engineering, vol. 8, no. 6, pp. 403-408, 2014.

[25] M. Turkyilmazoglu, "Flow of a micropolar fluid due to a porous stretching sheet and heat transfer," International Journal of NonLinear Mechanics, vol. 83, pp. 59-64, 2016.

[26] R. A. Mohamed and S. M. Abo-Dahab, "Influence of chemical reaction and thermal radiation on the heat and mass transfer in MHD micropolar flow over a vertical moving porous plate in a porous medium with heat generation," International Journal of Thermal Sciences, vol. 48, no. 9, pp. 1800-1813, 2009.

[27] K. Bhattacharyya, S. Mukhopadhyay, G. C. Layek, and I. Pop, "Effects of thermal radiation on micropolar fluid flow and heat transfer over a porous shrinking sheet," International Journal of Heat and Mass Transfer, vol. 55, no. 11-12, pp. 2945-2952, 2012.

[28] D. Prakash and M. Muthtamilselvan, "Effect of radiation on transient MHD flow of micropolar fluid between porous vertical channel with boundary conditions of the third kind," Ain Shams Engineering Journal, vol. 5, no. 4, pp. 1277-1286, 2014.
[29] Z. Ziabakhsh, G. Domairry, and H. Bararnia, "Analytical solution of non-Newtonian micropolar fluid flow with uniform suction/blowing and heat generation," Journal of the Taiwan Institute of Chemical Engineers, vol. 40, no. 4, pp. 443-451, 2009.

[30] A. A. Bakr, "Effects of chemical reaction on MHD free convection and mass transfer flow of a micropolar fluid with oscillatory plate velocity and constant heat source in a rotating frame of reference," Communications in Nonlinear Science and Numerical Simulation, vol. 16, no. 2, pp. 698-710, 2011.

[31] M. A. A. Mahmoud and S. E. Waheed, "MHD flow and heat transfer of a micropolar fluid over a stretching surface with heat generation (absorption) and slip velocity," Journal of the Egyptian Mathematical Society, vol. 20, no. 1, pp. 20-27, 2012.

[32] F. M. Abbasi, S. A. Shehzad, T. Hayat, and B. Ahmad, "Doubly stratified mixed convection flow of Maxwell nanofluid with heat generation/absorption," Journal of Magnetism and Magnetic Materials, vol. 404, pp. 159-165, 2016.

[33] B. Mliki, M. A. Abbassi, A. Omri, and B. Zeghmati, "Effects of nanoparticles Brownian motion in a linearly/sinusoidally heated cavity with MHD natural convection in the presence of uniform heat generation/absorption," Powder Technology, vol. 295, pp. 69-83, 2016.

[34] N. S. Elgazery, "The effects of chemical reaction, Hall and ion-slip currents on MHD flow with temperature dependent viscosity and thermal diffusivity," Communications in Nonlinear Science and Numerical Simulation, vol. 14, no. 4, pp. 1267-1283, 2009. 


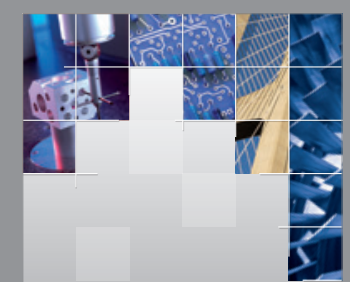

\section{Enfincering}
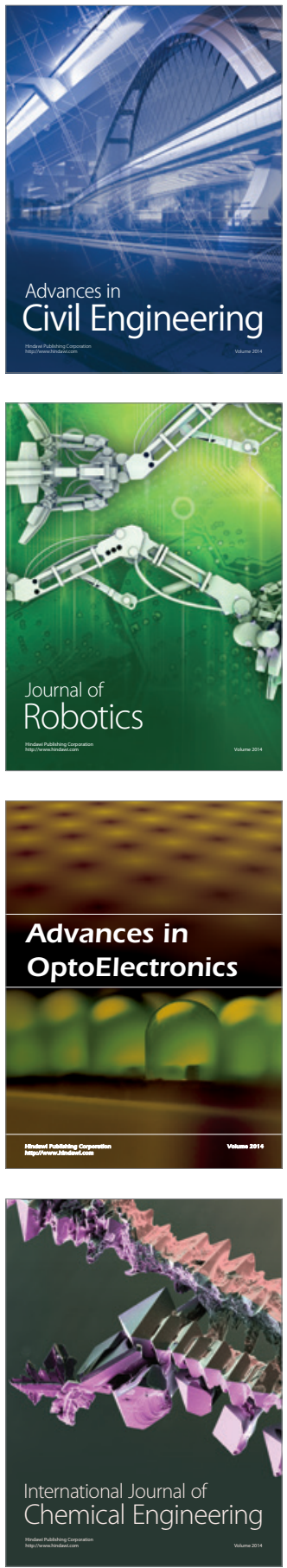

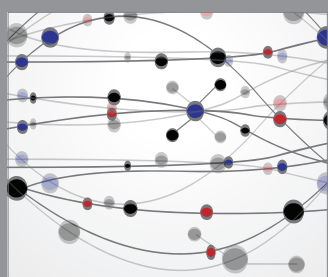

The Scientific World Journal

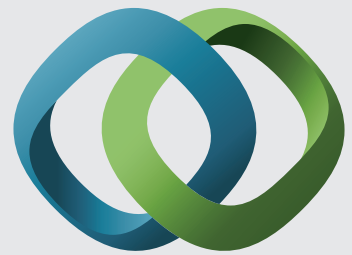

\section{Hindawi}

Submit your manuscripts at

http://www.hindawi.com
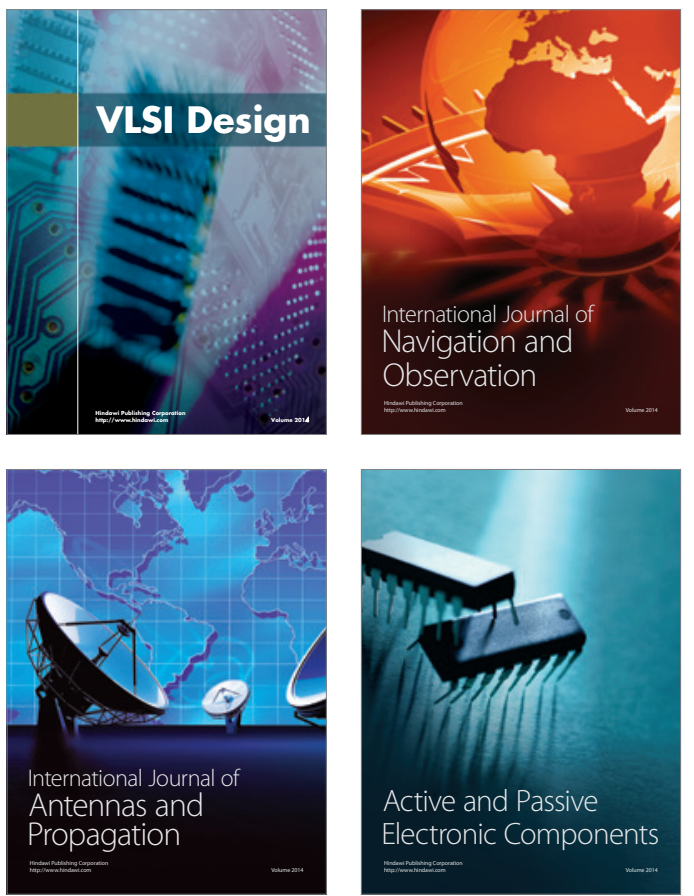
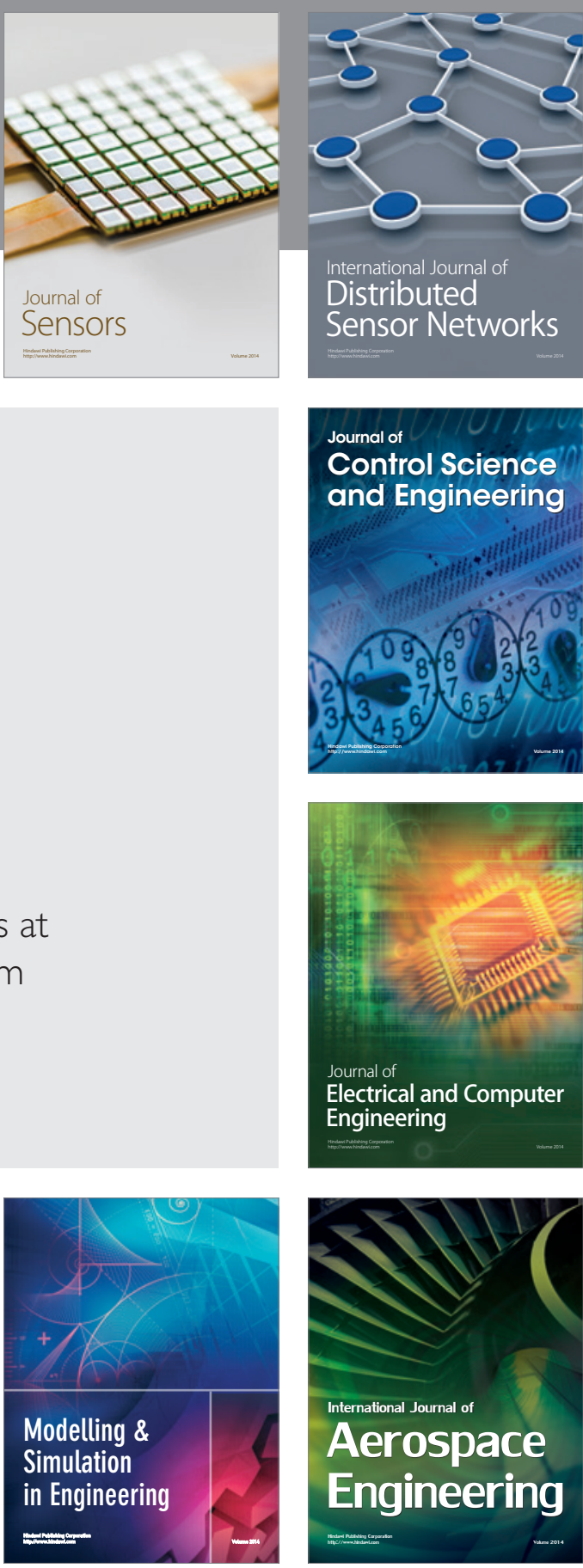

International Journal of

Distributed

Sensor Networks

Journal of

Control Science

and Engineering
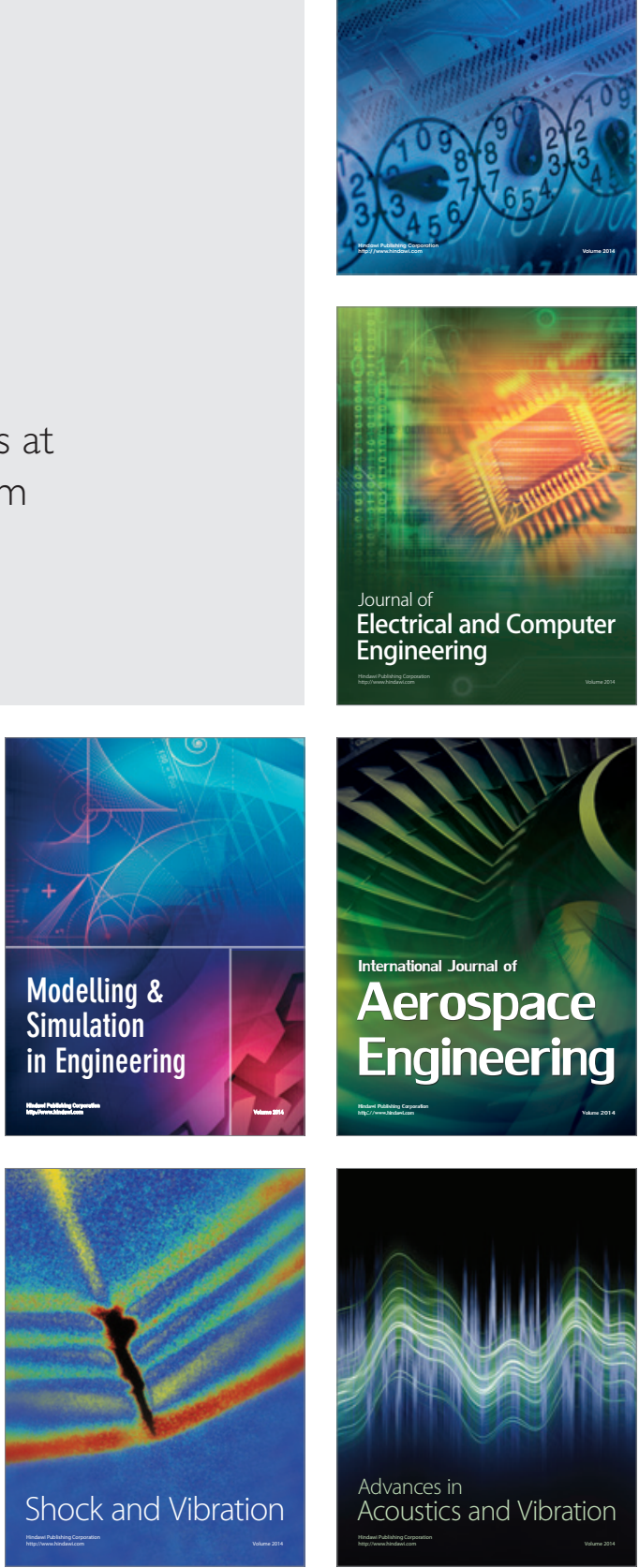